\title{
Réduction de frottement en écoulement turbulent par injection de macromolécules. Espoirs et déceptions *
}

\author{
Turbulent drag reduction by macromolecules injection \\ Hopes and desappointments *
}

\author{
D.H. Fruman \\ Laboratoire de biorhéologie \\ et d'hydrodynamique physiologique, \\ Université Paris VII
}

\author{
B. de Lagarde \\ Bertin \& Cie
}

\section{Introduction}

Depuis la découverte accidentelle par Toms $[1]^{* *}$ et Mysels [2] de la réduction de la résistance de frottement en régime turbulent, par addition de macromolécules solubles dans le fluide étudié, le phénomène qui porte le nom du premier a fait couler autant d'eau que d'encre. Pendant presque vingt ans, il resta dans une apparente léthargie et ne représenta qu'une curiosité de la mécanique des fluides, connue de quelques initiés. Ce ne fut qu'au début des années 60 qu'il fut redécouvert accidentellement par les pétroliers. A leur grande surprise, l'énergie nécessaire au pompage des boues de forage se trouvait considérablement diminuée par l'addition de polymères solubles à haut poids moléculaire. Ce qui n'était, dans le jargon dont on nous gratifie aujourd'hui, que la redécouverte et la mise au service de la production par un secteur socioéconomique aval d'une découverte fortuite de la recherche appliquée (puisque aussi bien dans le cas de Toms et de Mysels, il s'agissait d'étudier l'écoulement du Napalm), sonna le glas de l'oubli et ouvrit la voie à un puissant courant de recherche orientée et fondamentale. En effet, pour la première il s'agissait de prévoir des domaines d'application et de participer à l'effort d'innovation qui semblait prévisible ; tandis que pour la dernière il était question de fournir des informations concernant la caractérisation rhéologique des solutions diluées et semi-diluées de polymères et de permettre une interprétation, tout au moins phénoménologique, du processus de réduction de résistance de frottement. Il serait inutile de prétendre présenter dans un court exposé une revue exhaustive des résultats les plus marquants dans un domaine de recherche aussi vaste. Les

$\left(^{*}\right)$ Communication présentée au $17^{\mathrm{e}}$ Colloque d'aérodynamique appliquée, AAAF, Grenoble, novembre 1980.

$\left({ }^{* *}\right)$ Les nombres entre crochets renvoient à la bibliographie à la fin de l'article. lecteurs intéressés peuvent consulter les excellentes mises à jour de Hoyt $[3,4,5]$ et, en langue française, l'article de Deslouis [6]. Très modestement, nous allons nous limiter à considérer l'utilisation du phénomène de réduction de frottement par injection de corps macromoléculaires en hydrodynamique interne et externe. Plus particulièrement, on tachera de mettre en évidence les aboutissements présents par rapport aux espoirs passés et les perspectives futures eu égard aux déceptions actuelles.

Réduction de frottement : quelques chiffres

Il est évident que l'intérêt des chercheurs pour la réduction de la résistance de frottement tient principalement aux faibles quantités de polymère requises pour obtenir des effets considérables. En écoulement homogène, il suffit de quelques parts par million $\left(1 \mathrm{ppm}=10^{-6}\right)$ en poids de polymère (généralement des polyoxyethylènes, $\mathrm{PEO}$, ou des polyacrylamides, PAM) pour réduire, sous certaines conditions, de plus de moitié les pertes de charge en conduite. A titre d'exemple, la figure 1 montre des résultats obtenus par de Lagarde et al. [7] dans une conduite circulaire de $2 \mathrm{~mm}$ de diamètre. On observe que pour des concentrations croissantes la résistance de frottement augmente très rapidement jusqu'à atteindre un maximum de $75 \%$ pour à peu près $6 \mathrm{ppm}$ et diminue légèrement ensuite. Il en est de même pour les écoulements externes. Dans le cas d'une plaque plane [8], suffisamment mince de sorte que la presque totalité de la résistance soit due au frottement pariétal, une concentration de 0,125 ppm permet déjà de déceler des effets qui deviennent maximums et de l'ordre de $50 \%$ pour une concentration d'à peu près $4 \mathrm{ppm}$ (figure 2). Bien entendu, ces résultats ont été obtenus dans des installations d'essais de faible taille, conduites de petit diamètre et plaques courtes, et ne sont pas directement 


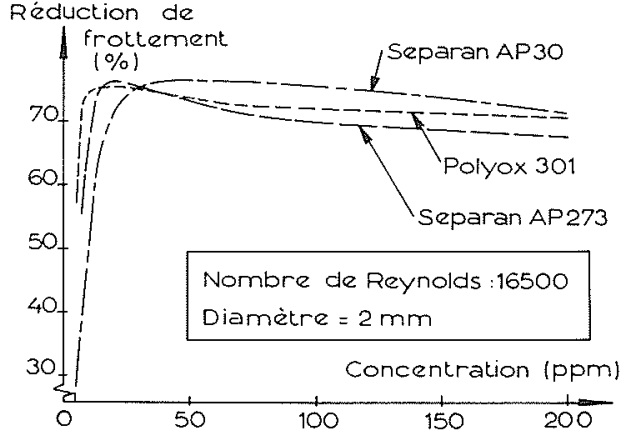

Figure 1 - Réduction de frottement dans une conduite de $2 \mathrm{~mm}$ de diamètre en fonction de la concentration des solutions de POLYOX WSR 301 (PEO) et SEPARAN AP 30 et AP 273 (PAM).

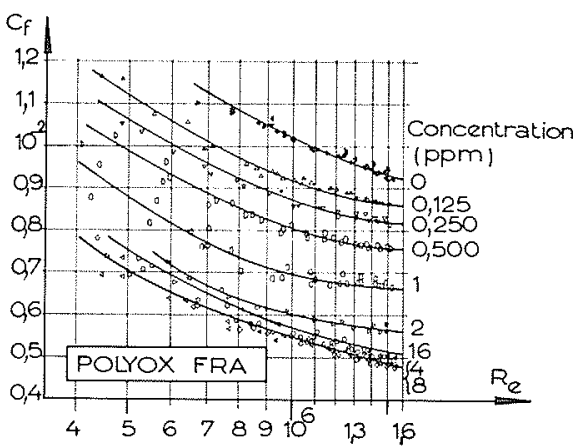

Figure 2 - Coefficient de résistance d'une plaque plane en fonction du nombre de Reynolds dans des solutions homogènes de POLYOX FRA, d'après [8].

extrapolables aux dimensions usuelles des applications industrielles et maritimes. En fait, leur extrapolation pose des problèmes ardus dont on donnera un aperçu par la suite.

Il est intéressant de s'interroger sur les changements qui interviennent au niveau de la structure même des écoulements avec réduction de résistance de frottement. Il est acquis que les molécules en solution modifient le profil des vitesses dans la zone proche de la paroi, souscouche visqueuse et de transition. Sans vouloir entrer trop dans les détails et en référant les lecteurs aux travaux de Virk [9] pour de plus amples informations, disons simplement que l'épaisseur de la sous-couche visqueuse se trouve prolongée par l'action des macromolécules et ceci d'autant plus que la réduction de la résistance de frottement est grande. On passe ainsi de la valeur courante de la sous couche visqueuse non dimensionnelle, de l'ordre de 10 dans le cas d'un fluide Newtonien, à des valeurs proches de 30 pour les solutions de polymères. Etant donné que pour les faibles concentrations en question la viscosité ne change pratiquement pas et que la vitesse de cisaillement diminue du fait de la réduction de frottement, l'épaisseur absolue de la sous couche visqueuse peut être plus de trois fois supérieure à celle du solvant. D’autre part, le phénomène ne se produit que si une vitesse de cisaillement critique est atteinte et le régime d'écoulement est turbulent.

\section{Injection de solutions de polymères : pourquoi ?}

Il est apparu assez rapidement que dans la plupart des applications pratiques envisageables, l'introduction du polymère par injection pariétale devenait nécessaire. Il suffit en effet de considérer quelques possibilités pour s'en apercevoir.

Dans le cas des applications maritimes la réponse est banale puisqu'il n'est pas question de rendre la mer réductrice de frottement. Par contre, c'est ici que le plus grand nombre d'occasions de se servir de la réduction de frottement semblait se présenter. En effet, quoi de plus attrayant que les corps de révolution dont la résistance de frottement est un pourcentage important de la résistance totale, ou que les engins non conventionnels (aéroglisseur à quille latérale ou hydroptères). dont la gourmandise en combustible pendant les périodes de veille (navigation sur coque) pourrait être réduite de façon à augmenter leur rayon d'action au cours des interventions, ou que les plans porteurs des hydroptères dont les gains modestes de résistance permettraient l'augmentation de leur vitesse ou de leur rayon d'action, ou que les hélices marines, dont le couple résistant pourrait être diminué, etc., pour envisager d'inonder de macromolécules cette zone proche de la paroi ou tout semble se passer ? Il ne faut pas oublier non plus le cas des navires de surface dont des essais au laboratoire et en mer ont montré la faisabilité technique des procédés d'injection.

Dans le cas du transfert de fluides, hydrodynamique interne, les applications semblaient multiples. Par exemple, pour le transport $d u$ brut où des injections judicieusement placées permettraient d'accroître la capacité des pipes en évitant d'augmenter leur diamètre ou le nombre de stations de pompage, ou, d'une manière générale, pour l'adaptation des installations d'adduction ou d'évacuation des usines à des augmentations temporaires ou permanentes de leur capacité. Rappelons que, dès la fin des années 60 , les pompiers de New York utilisaient les polymères pour augmenter la portée de leurs lances à incendie grâce à la diminution des pertes de charge obtenues dans les tuyauteries souples. On pourrait aussi envisager de rendre des égouts capables d'évacuer des débits supérieurs à leur capacité nominale de dimensionnement en asservissant les injections de polymères à ceux-ci. Enfin, les portes restaient ouvertes pour accueillir des problèmes encore plus ponctuels tels que celui du transfert des mélanges solide-liquide (slurries) sur des longues distances ou, à l'opposé, les applications médicales liées à l'hémodynamique.

L'éventail était donc vaste et les possibilités étaient énormes. Sans prétendre que la réduction de frottement deviendrait un fait courant, on pouvait regarder l'avenir avec un optimisme non dénué de fondement.

Dans ce qui suit, nous aborderons quelques uns des problèmes qui ont retardé la mise en application du procédé en dépit d'un transfert de connaissance, du laboratoire à l'industrie, assez efficace. 


\section{Diffusion des solutions de polymères : plaques planes}

Ayant reconnu l'intérêt que pouvait présenter l'utilisation des propriétés réductrices de frottement en écoulement externe, il s'agit de savoir comment les solutions peuvent être introduites dans la zone pariétale et, surtout, de connaitre les lois qui régissent le processus de diffusion de celles-ci. Nous ne nous adresserons pas au problème technologique qui consiste à concevoir des mélangeurs automatisés capables de produire ces solutions concentrées avec une grande fiabilité ou à celui de la définition géométrique et hydrodynamique des injecteurs. Disons néanmoins, qu'en ce qui concerne les mélangeurs, des réalisations industrielles existent en France [10] et à l'étranger [11]. Pour ce qui est des injecteurs il est possible d'envisager plusieurs solutions : distribués en utilisant des matériaux poreux [12], ou ponctuels au moyen de fentes minces [13] ou de plaques perforées [14].

Dans ce qui suit, nous allons revoir quelques résultats concernant la diffusion des solutions de polymères et, en particulier, les formulations empiriques qui permettent d'estimer les concentrations pariétales dans une zone éloignée des injecteurs. Rappelons brièvement que les études de Wells et Spangler [12] ont montré que la résistance de frottement diminue si les polymères occupent la couche pariétale de fluide à l'intérieur de la limite supérieure de la couche intermédiaire ou de transition (buffer layer). Wu [15] et Wu et Tulin [16] ont introduit la notion de débit de sous couche visqueuse, $Q_{v}$, et ont montré que les débits d'injection optimums doivent être comparables à celuici, donné par

$$
Q_{v}=\frac{1}{2} \delta_{v} u_{v}=\frac{1}{2} \delta_{v} u^{*} \bar{\delta}_{v}=\frac{1}{2} \bar{\delta}_{\nu}^{2} \nu
$$

où $\delta_{v}$ et $u_{v}$ sont respectivement l'épaisseur et la vitesse à la limite extérieure de la sous couche visqueuse, $\nu$ est la viscosité du fluide, $\delta_{\nu}=\delta_{v} u^{*} / \nu$, et $u^{*}$ la vitesse de cisaillement à la paroi. Remarquons que $Q_{\nu}$ dépend sculement de la viscosité du fluide puisque $\delta_{v}$ est une constante. Dans le cas idéal, il suffirait d'assurer un débit égal à celui de la sous couche visqueuse, épaissie en présence de polymères, avec une concentration d'additif proche de l'optimum $(\sim 10 \mathrm{ppm}$ pour les PEO et les PAM). S'il s'agit d'une injection pontuelle, il faudrait connaître la concentration pariétale le long de la plaque et s'assurer qu'elle ne soit pas trop faible par rapport à l'optimale au bord de fuite de l'objet considéré ou au droit d'une nouvelle fente d'injection. On est donc tenu de connaître la fonction reliant les concentrations pariétales aux paramètres caractéristiques de l'injection et à la distance à l'injecteur.

La concentration pariétale d'un traceur inerte contenu dans un fluide éjecté ponctuellement et tangentiellement à une paroi solide sur laquelle existe une couche limite turbulente est déterminée, au voisinage de la source, par une zone initiale où la diffusion moléculaire est prépondérante. Cette zone est suivie par une zone de transition dont nous en négligerons l'effet dans cette discussion et une zone finale où la diffusion est de nature turbulente. Dans la zone initiale, la concen- tration pariétale ne change que très peu avec la distance, tandis que dans la zone finale, elle évolue très vite avec celle-ci.

Wu [17] avait constaté, en mesurant la concentration loin de l'injecteur dans un point situé à l'intérieur de la couche limite d'une plaque plane, que celle-ci était beaucoup plus élevée dans le cas de l'injection de poly. mère que de celle d'eau. Il en avait déduit que le trans. port turbulent était sensiblement diminué du fait même de l'interaction des solutions de polymères avec l'écoulement externe. Des expériences complémentaires avec des jets de polymère dans un écoulement turbulent derrière une grille [15] semblaient confirmer cette hypothèse.

Cependant, des expériences $[18,19,20]$ portant sur la mesure des concentrations le long de plaques planes au cours de l'injection de solutions de polymères ont, ultérieurement, modifié cette hypothèse. Les résultats ont montré qu'il ne s'agit pas d'un phénomène de diminution de la diffusion turbulente, mais plutôt d'un allongement substantiel de la zone initiale où les phénomènes de diffusion moléculaire sont prédominants. La figure 3 montre des résultats obtenus par Fruman et Tulin [21] pour l'injection, à égale vitesse, d'eau et de solutions de 500 et 1000 ppm de Polyox WSR 301 . On observe très nettement le prolongement de la zone initiale et la rapide décroissance dans la zone finale.

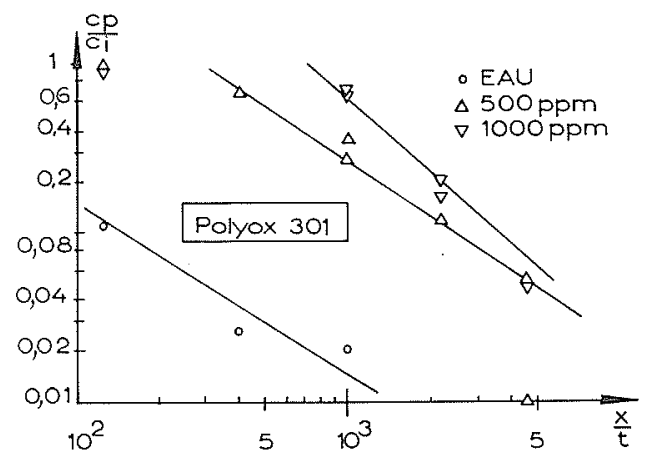

Figure 3 -Concentration relative de POLYOX WSR 301 le long d'une plaque plane, d'après [21].

Les valeurs numériques données par Collins et Gorton [20] pour des injections de PEO ont été portées sur la figure 4 en utilisant comme abscisse réduite $V \cdot x / q_{i}$ où $q_{i}$ est le débit de la source. Ici aussi, on note la très nette influence du polymère sur la longueur de la zone initiale. Par ailleurs, les résultats montrent que pour l'injection d'une solution de $2000 \mathrm{ppm}$, les concentrations à la paroi n'augmentent pas comme on aurait pu le prévoir en extrapolant les résultats obtenus pour 500 et $1000 \mathrm{ppm}$. Remarquons finalement que le débit maximum de la source était dans [20] égal au débit de la sous couche visqueuse pour une vitesse extérieure de $0,8 \mathrm{~m} / \mathrm{s}$ et une plaque de $0,53 \mathrm{~m}$, tandis qu'en [18] il était entre 20 et 30 fois supérieur pour une vitesse extérieure de $10,65 \mathrm{~m} / \mathrm{s}$ et une plaque de $3 \mathrm{~m}$ de long. En dépit de ces différences subtantielles, les caractéristiques générales du phénomène de diffusion des poly. mères sont très similaires dans les deux cas. Fruman et Tulin [18] ont déduit que pour des concentrations éjectées de Polyox WSR 301 comprises entre 100 et 


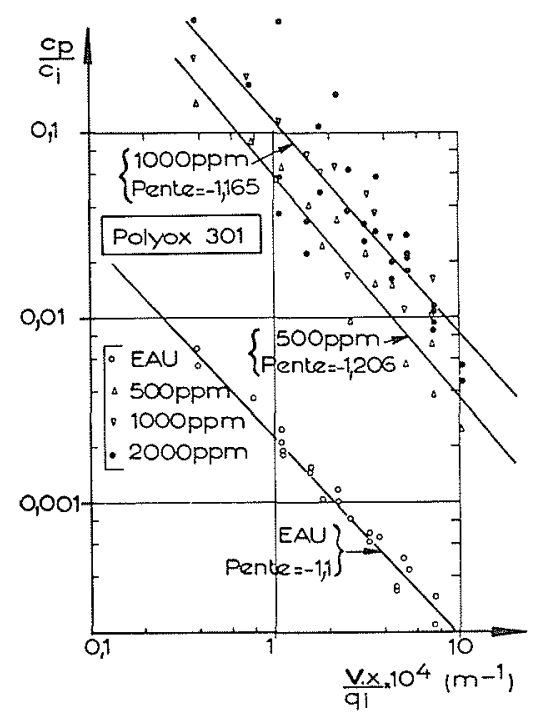

Figure 4 - Concentration relative de $\mathrm{PEO}$ le long d'une plaque plane, d'après [20].

1000 ppm la longueur de la zone initiale $L_{i}$ pouvait être estimée par :

$$
L_{i}=7,2\left(\frac{v_{i}}{V}\right)^{1,5} t c_{i}
$$

où $c_{i}$ et $v_{i}$ sont respectivement la concentration et la vitesse d'injection, $t$ est l'épaisseur de la fente d'injection et $V$ est la vitesse de l'écoulement externe. Un calcul rapide montre que pour des concentrations de 100 et 1000 ppm et $v_{i} / V=0,3$ les longueurs initiales sont respectivement de 65 et 650 fois $t$, soit bien supérieures à celles observées dans le cas d'une injection d'eau, où $L \simeq 10 t$. Ces ordres de grandeur de l'allongement de la zone initiale ont été justifiés par une analyse [18] qui tient compte de l'épaississement de la sous couche visqueuse et de la diminution $\mathrm{du}$ coefficient de diffusion moléculaire des solutions de polymères à forte concentration.

Dans la zone finale, $L>L_{i}$, la concentration pariétale, $C_{p}$, est donnée par [18]

$$
C_{p}(L)=10,79\left(\frac{v_{i}}{V}\right)^{1,74}\left(\frac{t}{L}\right)^{1,16} C_{i}^{2,16}
$$

où l'on voit qu'elle diminue plus rapidement que pour un fluide newtonien pour lequel la variation de $C_{p}$ est proportionnelle à l'inverse de la distance à une puissance inférieure à l'unité et comprise, suivant les auteurs $[18,22,23]$, entre 0,71 et 0,9 . Pour un polymère acrylique, le Reten 423, Latto et El Riedy [19] ont observé aussi une augmentation de la diffusivité turbulente relative à celle de l'eau. La figure 5 montre l'ensemble des résultats expérimentaux de Fruman et Tulin [18] et la distinction assez nette entre les deux zones de diffusion considérées.

Avant de clore cette section, on doit faire remarquer que toutes les études citées restent partielles, soit parce que les expériences ont été conduites avec une seule vitesse de l'écoulement externe, soit parce que les dimensions des plaques utilisées sont différentes, soit parce que l'on a employé des polymères différents, etc.

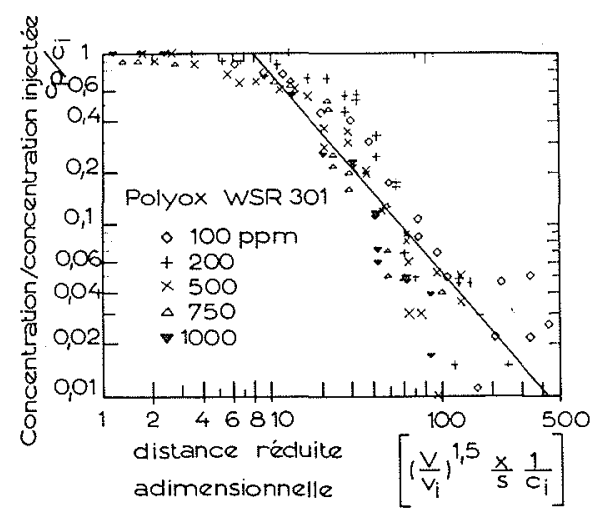

Figure 5 - Concentration relative de polymère en fonction de la distance réduite. Ensemble des résultats d'après $[18,21]$.

Ceci rend la comparaison, l'interprétation et l'extrapo. lation des résultats particulièrement difficile et délicate.

\section{Diffusion et réduction de frottement : plaques planes}

A notre connaissance, un seul travail expérimental (Fruman et Tulin [18]) a permis de déterminer simultanément les effets de réduction de frottement sur une plaque plane et l'évolution des concentrations pariétales le long de celle ci ; toutes les autres recherches se sont adressées à l'un ou l'autre de ces problèmes indépendamment. Pour fixer les idées, la figure 6 montre la réduction de la résistance de frottement sur une plaque plane de $3 \mathrm{~m}$ de longueur en fonction de la concentration mesurée à une distance de $2,37 \mathrm{~m}$ de l'injecteur et de $0,48 \mathrm{~m}$ en amont du bord de fuite. On observe que pour des concentrations éjectées inférieures ou égales à $500 \mathrm{ppm}$, la réduction de frottement augmente et, en dépit d'une certaine dispersion des résultats, atteint presque $60 \%$ pour des concentrations pariétales de l'ordre de quelques dizaines de ppm. Pour des concentrations supérieures, 750 et $1000 \mathrm{ppm}$, l'efficacité de l'injection diminue même si la concentration pariétale augmente. Cet effet est encore plus notable si l'on utilise la concentration au bord de fuite calculée en utilisant l'expression (3) (figure 7).

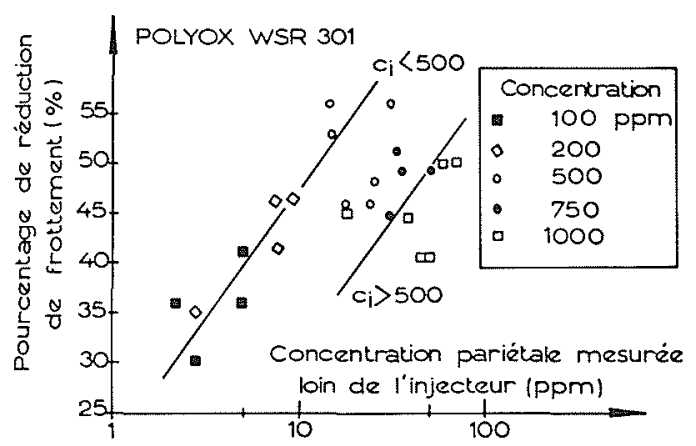

Figure 6 - Réduction de frottement en fonction de la concentration de polymère mesurée près du bord de fuite d'une plaque plane, d'après [21]. 


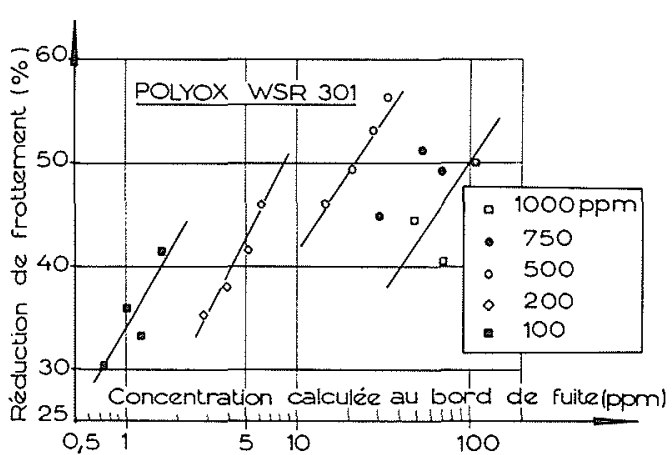

Figure 7 - Réduction de frottement en fonction de la concentration de polymère calculée au bord de fuite d'une plaque plane, d'après [18].

De Lagarde [24] a utilisé le modele de Fruman et Tulin pour correler d'une manière semblable les réductions de frottement mesurées sur une plaque plane pesée indépendamment de l'injecteur (ce qui n'était pas le cas pour [18]). Quelques-uns de ces résultats sont donnés sur les figures 8,9 et 10 .

Pour la première, il s'agit d'une fente très mince, de $0,2 \mathrm{~mm}$ d'épaisseur. Comme dans la figure 7 , obtenue avec un injecteur de $0,5 \mathrm{~mm}$, on observe une diminution du rendement, en terme de réduction de frottement,

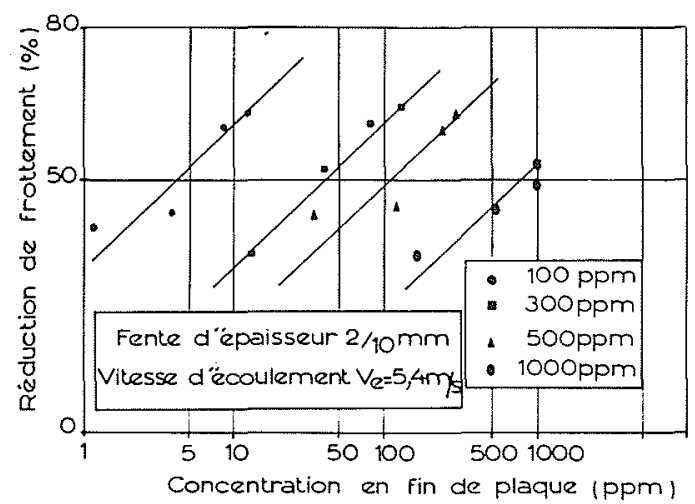

Figure 8 - Réduction de frottement en fonction de la concentration de polymère calculée au bord de fuite d'une plaque plane, d'après [24]. Injection par fente mince, $0,2 \mathrm{~mm}$.

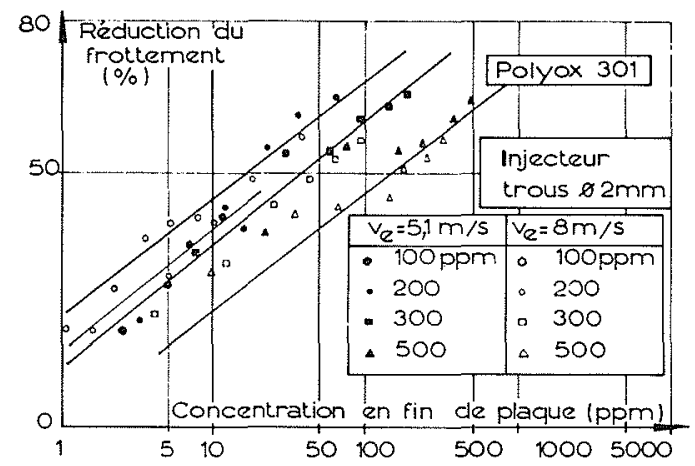

Figure 9 - Réduction de frottement en fonction de la concentration de polymère calculée au bord de fuite d'une plaque plane, d'après [24]. Injection par paroi perforé, $\emptyset=2 \mathrm{~mm}$, fente équivalente $0,5 \mathrm{~mm}$.

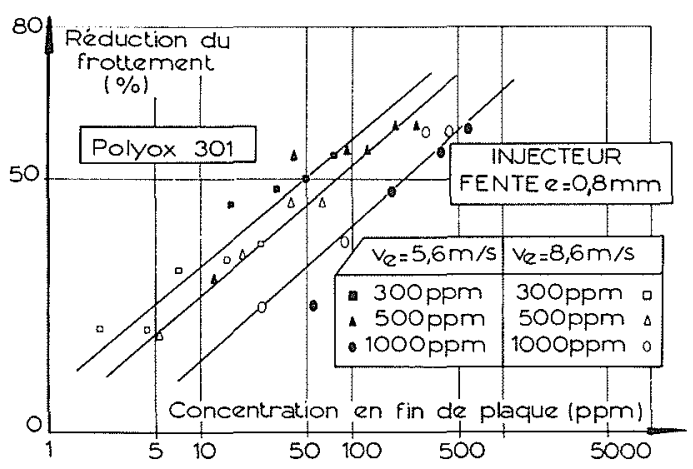

Figure 10 - Réduction de frottement en fonction de la concentration de polymère calculée au bord de fuite d'une plaque plane, d'après [24]. Injection par fente mince, $0,8 \mathrm{~mm}$.

au fur et à mesure que la concentration injectée augmente. $\mathrm{Si}$ au lieu d'une fente on utilise une paroi perforée, équi. valente à une fente de $0,5 \mathrm{~mm}$, le phénomène reste à peu près similaire sauf en ce qui concerne la plus faible des concentrations, 100 ppm, qui semble avoir été soumise à une certaine dégradation au passage des orifices d'injection. Pour une fente de $0,8 \mathrm{~mm}$, figure 10 , le rendement d'une injection de $1000 \mathrm{ppm}$ est plus grand que pour la fente de $0,2 \mathrm{~mm}$, indiquant ainsi un effet de l'épaisseur de la fente aux fortes concentrations.

Il apparait donc que l'augmentation de la concentration injectée n'apporte pas, pour une plaque de longueur donnée, une amélioration des performances et qu'au contraire, à consommation de polymère égale, elle entraine une dégradation de celles-ci. Dans la figure 11, nous avons porté des résultats de réduction de frottement présentés en [25] en fonction de la concentration moyenne le long de la plaque calculée en utilisant le modèle de diffusion déjà mentionné. On observe que même pour des concentrations moyennes assez faibles, inférieures à $10 \mathrm{ppm}$ (ce qui semble indiquer une assez bonne dilution du polymère), la réduction de frottement est sévèrement limitée par la concentration de la solution injectée.

Deux explications peuvent être avancées pour interpréter ce phénomène qui avait déjà été mentionné il y a fort longtemps [26]. D'une part, l'allongement notable de la zone initiale entraine nécessairement une diminu-

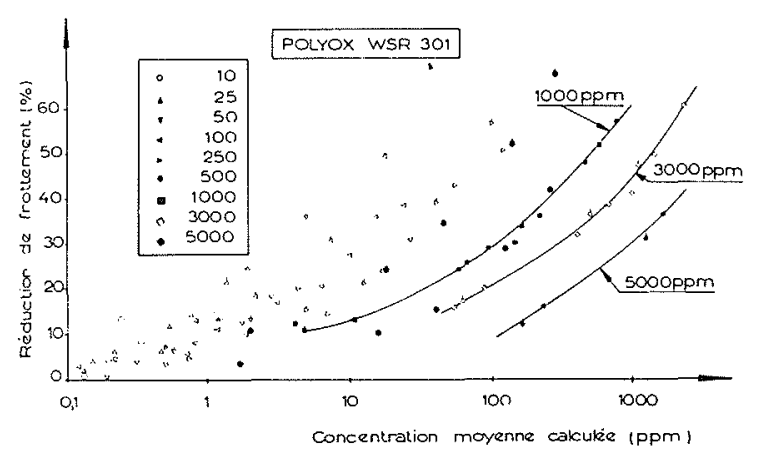

Figure 11 - Réduction de frottement en fonction de la concentration moyenne calculée le long d'une plaque plane, d'après [25]; 
tion locale des effets réducteurs de résistance de la solution de polymères. Ceci a déjà été constaté dans le cas de plaques planes en fluide homogène de polymère, voir [15] par exemple. D'autre part, des études récentes [27, 28] ont montré que la zone proche en aval des injecteurs est, au cours de l'injection de solutions de polymères, le siège de phénomènes viscoélastiques liés à la relaxation des contraintes normales des solutions. On a pu mettre en évidence des effets du gonflement (épaississement) des jets qui sont responsables de la déviation de l'écoulement externe même aux grandes vitesses. Par ailleurs, malgré le cisaillement considérable entre la couche épaissie de polymère et l'écoulement externe, on n'observe pas une dilution rapide de la solution éjectée. Bien que l'on en soit encore réduit aux hypothèses, on peut avancer que le phénomène d'épaississement du jet et d'entraînement de la couche de polymère épaissie (et donc décelérée) s'accompagne d'une dissipation d'énergie complémentaire qui vient diminuer l'efficacité globale de l'injection de polymère.

D'autres problèmes restent posés en ce qui concerne la dégradation des solutions de polymères aux vitesses élevées de l'écoulement externe. Il est en effet probable que, soumises à des taux de cisaillement (déformation) importants, les molécules perdent de leur efficacité et diminuent le rendement de l'injection.

On voit donc que dans le cas des écoulements externes, il reste encore beaucoup à faire avant d'aboutir à une compréhension du phénomène permettant d'aborder les applications pratiques avec des chances de succès.

\section{Diffusion et réduction de frottement : conduites}

Il est évident que ce qui vient d'être discuté dans les sections précédentes est applicable dans le cas des écoulements dans les conduites et vice versa. Néanmoins, du point de vue pratique, le problème est fondamentalement différent puisque dans les canalisations la longueur de la zone de mélange sera relativement courte par rapport à leur longueur totale. En dehors de cette zone de mélange, il faudra simplement considérer les résultats obtenus pour des solutions homogènes.

Il est néanmoins instructif de considérer, avant d'aborder d'autres problèmes, des résultats récents obtenus par Fruman et Galivel [27, 28]. Au cours d'essais effectués avec un injecteur faiblement incliné par rapport à la paroi d'une veine rectangulaire, ils ont mis en évidence des changements notables de pressions pariétales en aval de l'injecteur pendant l'introduction de solutions de polymères. La figure 12 présente quelques uns de leurs résultats montrant l'évolution des pressions statiques en fonction de la distance à l'injecteur. Au voisinage de celui-ci, les pressions diminuent très rapidement quand on injecte la solution de polymère. Cette diminution de pression n'est pas une perte de charge puisqu'elle est suivie d'une zone à gradient de pression positif indiquant une recompression de l'écoulement. La réduction de frottement, assimilée au changement de pente par rapport à celle de l'écoulement avec injection d'eau, n'est pas notable, pour la concentration de 2000 ppm de Polyox WSR 301 considérée, sur une longueur relativement importante en aval de cette zone. Ceci est

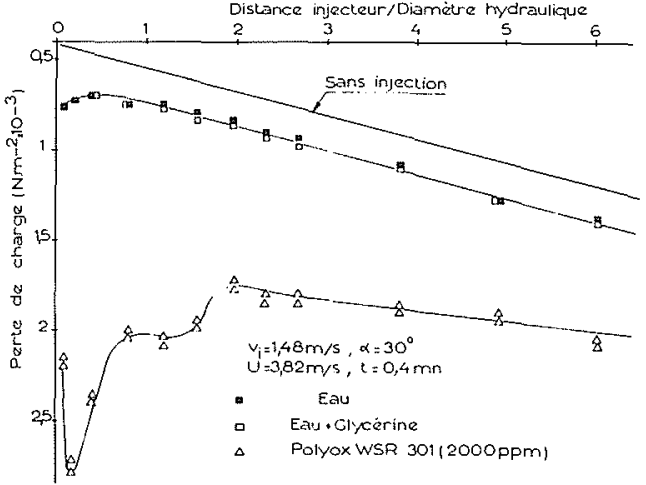

Figure 12 - Distribution des pressions statiques dans un écoulement en conduite sans injection et avec injection d'eau et d'une solution de polymère, d'après [27]

une indication supplémentaire de la très faible dilution du polymère déjà mentionnée dans le cas des plaques planes. En outre, ces résultats mettent en évidence l'existence de phénomènes de nature viscoélastique dans la région proche de l'injecteur.

En effet, des visualisations de l'écoulement ont permis de montrer que les diminutions des pressions statiques sont dues à un rapide épaississement des jets de polymères dès leur sortie des fentes d'injection. Il en résulte une décélération de la couche de polymère et une accélération de l'écoulement externe à la suite du rétrécissement de l'épaisseur effective de la veine d'essais. La couche de polymère épaissie se comporte vis-à-vis de l'écoulement externe comme une paroi presque solide ainsi qu'une analyse des résultats expérimentaux a permis de le montrer [27]. Par ailleurs, ce comportement aboutit à une dissipation d'énergie beaucoup plus grande que dans le cas des injections d'eau $[13,14]$. Bien que le phénomène décrit soit d'une importance considérable dans le cas des écoulements externes, il n'a qu'un impact limité dans les applications industrielles au transport en conduite. Dans ce dernier cas, il ne s'agit que d'un effet très localisé par rapport à la longueur totale de celle-ci. D'autres problèmes, tels que ceux liés à l'effet de diamètre et de la dégradation, sont alors prédominants et discutés ci-dessous.

Dès les premiers essais effectués avec des conduites de diamètre différent, on avait pu mettre en évidence un effet de diminution de l'efficacité des solutions de polymère au fur et à mesure que le diamètre augmentait, et ceci, aussi bien à égalité des nombres de Reynolds que des vitesses. La figure 13, d'après la référence [29], montre que pour une conduite de $254 \mathrm{~mm}$, la réduction de frottement maximale n'est que de $38 \%$, bien inférieure à celle observée dans des tubes de très petit diametre (figure 1). D'autre part, aucune réduction de frottement n'apparait avant d'avoir atteint des nombres de Reynolds de l'ordre de 60000 , bien supérieurs aux seuils mis en évidence dans les petits diamètres. Ce type de comportement est directement lié à l'existence d'une vitesse de cisaillement critique, $u_{c r}^{*}$, en deça de laquelle les macromolécules ne sont pas efficaces. Une analyse détaillée du problème ainsi qu'une méthode semi-empirique d'extrapolation des résultats a été présenté suite à des études menées à bien par Bertin \& Cie [30]. La mé- 


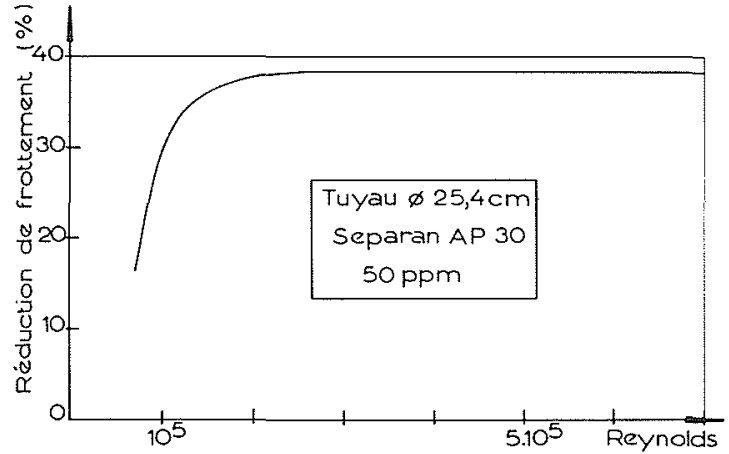

Figure 13 - Réduction de frottement en fonction du nombre de Reynolds pour une solution de PEO dans une conduite de $254 \mathrm{~mm}$, d'après [29].

thode a été vérifiée expérimentalement, utilisant des résultats obtenus avec des solutions de Gomme de Guar, un polymère naturel extrêmement résistant à la dégradation mécanique mais beaucoup moins efficace que les PEO et les PAM, dans des conduites de différents diamètres compris entre $4,1 \mathrm{~mm}$ et $208 \mathrm{~mm}$. La figure 14 montre l'effet de diamètre à vitesse d'écoulement constante. En utilisant le modèle semi-empirique proposé en [30] le maximum de réduction de résistance de frottement pour le Polyox WSR 301 a été évalué pour une conduite de $500 \mathrm{~mm}$ de diamètre (figure 15). Pour atteindre des valeurs de l'ordre de $70 \%$, il faudrait utiliser des concentrations de l'ordre de $100 \mathrm{ppm}$, plus de dix fois supérieures á celles obtenues expérimentalement dans une conduite de $2 \mathrm{~mm}$ (figure 1).

L'augmentation de la concentration associée à l'effet de diamètre ne serait pas "a priori" rédhibitoire économiquement et techniquement s'il ne venait pas se greffer, dans le cas des polymères à très hauts poids moléculaires, le problème de la dégradation mécanique des solutions. Parmi les multiples études consacrées à ce problème, Fruman et al. [31] ont montré que le frottement pariétal le long d'une conduite n'atteint un régime établi que si les contraintes de cisaillement ne dépassent pas des valeurs critiques correspondantes à la dégradation mécanique des macromolécules en solution. Au-delà de ces contraintes critiques, l'écoulement n'est pas nécessairement établi, dans le sens que le frottement local augmente avec la distance comme il est montré dans la figure 16. La dégradation ne se produit pas seulement

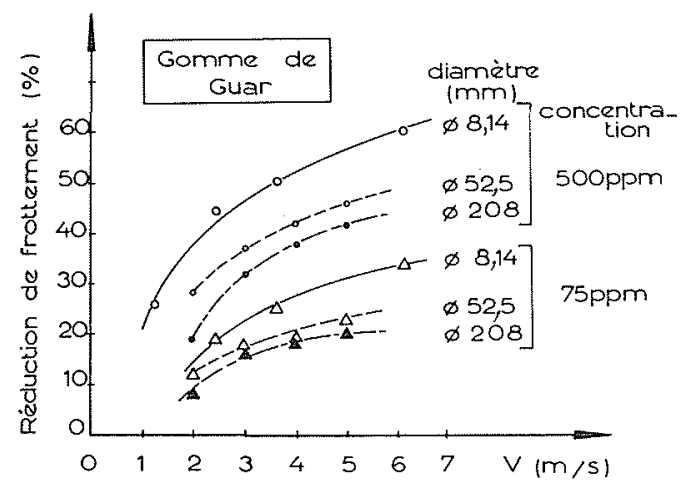

Figure 14 - Réduction de frottement en fonction de la vitesse pour des solutions de Gomme de Guar dans trois conduites de diamètre différent, d'après [30].

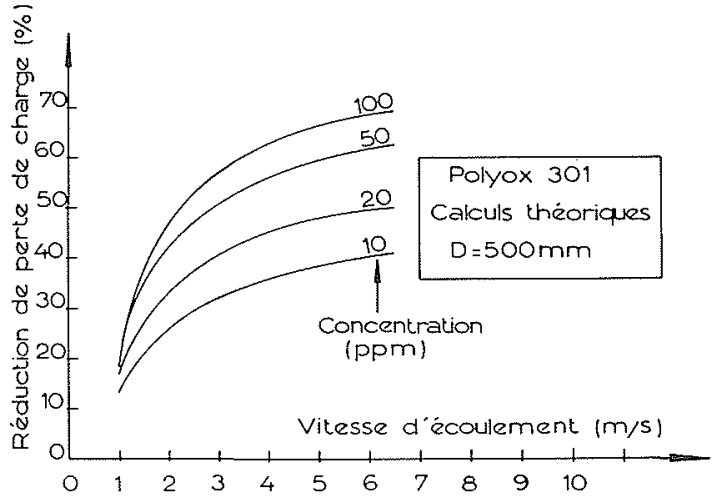

Figure 15 - Maximum de réduction de frottement attendue en fonction de la vitesse de l'écoulement pour des solutions de POLYOX WSR 301, d'après [30].

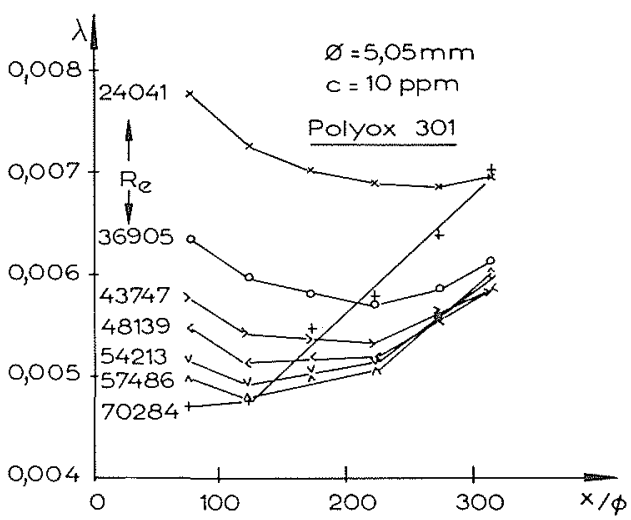

Figure 16 - Réduction de frottement locale en fonction de la distance ; effet de la dégradation, d'après [31].

dans les conduites, mais aussi dans les pompes et dans tous les accidents hydrauliques, vannes, contractions, etc... du circuit. Il serait long et fastidieux de rentrer dans le détail des études effectuées sur ce sujet. Nous dirons seulement que la dégradation mécanique des solutions de polymères a été un facteur déterminant dans le retard pris par les applications industrielles du procédé. C'est probablement dans ce domaine que des efforts de recherche sont nécessaires si l'on veut disposer des macromolécules très efficaces et très résistantes.

\section{Exemples d'applications}

Comme dans toute recherche du type fondamental, le passage aux applications doit répondre à des motivations fort spécifiques, surtout quand, comme dans le cas présent, elle ne s'adresse pas nécessairement à la satisfaction immédiate d'un besoin. Les motivations peuvent être de trois types: stratégiques quand il s'agit des problèmes répondant à des questions de sécurité ou de prestige, technologiques quand le choix d'autres solutions est limité ou inexistant, et économiques quand le procédé apporte un gain appréciable de coûts, fixes ou d'exploitation, par rapport à d'autres possibilités. La situation idéale est celle, bien entendu, où ces trois motivations se trouvent réunies et satisfaites simultanément. Nous devons reconnaître que dans le cas de la réduction de frottement, on n'a pu répondre qu'à quel- 
ques problèmes ponctuels dont nous tacherons de donner un aperçu rapide.

Le cas qui nous semble le plus intéressant est celui du transport de pétrole brut. Cette application fut envisagée dès le tout début des recherches mais on s'est vite aperçu que les gains, quand ils existaient, étaient marginaux dans le cas des huiles légères et des oléoducs destinés à absorber des débits pratiquement constants pendant de longues années. Il a donc fallu attendre jusqu'à la récente mise en exploitation des gisements de l'Alaska pour trouver des motivations technologiques et économiques justifiant la mise en œuvre du procédé. Il s'agissait de choisir entre l'installation de stations de pompage relais capables d'augmenter la capacité d'un "pipe" de 1,20 m de diamètre et plusieurs centaines de kilomètres de longueur pour absorber un pic de production de courte durée, à peu près deux ans, ou d'utiliser les solutions de polymère pour réduire les pertes de charge et assurer un plus grand débit avec les installations de pompage existantes. Le choix était donc économique d'une part: coût et amortissement des installations fixes nouvelles contre coûts d'opération, et technologique d'autre part: unités d'injection simples contre lourds systèmes de pompage. La balance pencha, pour une fois, vers l'injection de solutions de polymères [32]. Cette expérience pilote est en opération depuis plus d'un an et semble satisfaire les utilisateurs.

Dans le même ordre d'idées, on peut signaler aussi les expériences pilotes menées à bien en France et à l'Etranger pour augmenter la capacité des égouts. Dans bien des cas, il s'avère, à la suite des besoins nouveaux créés par l'urbanisation, qu'ils deviennent inadaptés. Etant donné les marges de sécurités observées dans la réalisation des installations anciennes, leur capacité d'absorption des incréments de débit est assez grande et n'est inadéquate que pour des durées d'utilisation relativement courtes. Le choix d'une installation d'injection de polymères asservie aux débits (ou aux niveaux) est alors technologiquement et économiquement rentable. Des augmentations de vitesse de $70 \%$ avec une injection correspondante à $50 \mathrm{ppm}$ de Polyox WSR 301 dans l'écoulement ont été obtenues [11].

Des études menées en France [35] sur un collecteur de $0,9 \mathrm{~m}$ de diamètre et de $3 \%$ de pente rempli à $15 \%$, ont permis de mettre en évidence, simultanément : une diminution du tirant d'eau de $10 \%$ et une augmentation de vitesse moyenne de $58 \%$ avec une consommation de polymère équivalente à une concentration homogène de $37 \mathrm{ppm}$. La réduction de la résistance de frottement est comprise entre 50 et $75 \%$.

Signalons aussi les expériences conduites pour augmenter la capacité des pipelines transportant, au bassin de décantation, les cendres provenant des laveurs de fumée des centrales thermiques. L'augmentation de la production entraine une augmentation du débit de ces suspensions et donc la nécessité de construire une nouvelle conduite ou une nouvelle station de pompage. Les polymères apportent ici une solution d'autant plus intéressante que les longueurs sont relativement courtes et les problèmes de dégradation des solutions acqueuses se posent à un degré moindre [34].

Dans le domaine que l'on pourrait classer comme stratégique, l'utilisation des polymères pour augmenter la
Tableau 1. Amélioration des débits et des portées d'une lance à incendie

\begin{tabular}{|c|c|c|c|c|c|}
\hline & $\begin{array}{c}\text { Cuyau } \\
\mathrm{mm}\end{array}$ & $\begin{array}{c}\text { Pression } \\
\text { refoule } \\
\text { ment } \\
\text { Pompe } \\
\text { bar }\end{array}$ & $\begin{array}{c}\text { Pression } \\
\text { lance } \\
\text { bar }\end{array}$ & $\begin{array}{c}\text { Débit } \\
1 / \mathrm{mn}\end{array}$ & $\begin{array}{c}\text { Longueur } \\
\mathrm{m}\end{array}$ \\
\hline $\begin{array}{c}\text { avec } \\
\text { O'GLISS }\end{array}$ & 45 & 19 & 8,7 & 610 & 260 \\
\hline eau & 70 & 18 & 3,6 & 845 & 600 \\
\hline $\begin{array}{c}\text { aVec } \\
\text { O'GLISS }\end{array}$ & 70 & 18 & 4,9 & 1280 & 600 \\
\hline
\end{tabular}

portée des lances à incendie est un bon exemple. Le système O'Gliss de la Société Bertin \& Cie a été essayé avec succès sur un prototype industriel [35]. Le tableau I montre les augmentations substantielles de débit et portée d'une lance alimentée avec une solution de l'ordre de 50 ppm de Polyox WSR 301 par rapport à une autre avec de l'eau.

Le domaine stratégique ne serait pas complet sans une mention des applications militaires. Parmi celles publiées, notons les essais sur un dragueur de mines, effectués par la Royal Navy [36] en utilisant une technique d'injection ponctuelle. Bien que les résultats indiquaient des réductions de résistance de frottement appréciables (22 à $36 \%)$, il ne semble pas que ces efforts, déjà anciens puisqu'ils remontent à 1969 , aient eu de suite. Il faut dire qu'à l'époque, le rapport entre le coût du polymère nécessaire et le coût de combustible économisé était de l'ordre de 1500 , ce qui enlevait tout intérêt économique à la méthode. Par ailleurs, le poids de polymère à transporter était bien supérieur à celui du fuel. On voit mal quelle pourrait être la découverte qui permettrait, de nos jours, de réduire ces handicaps. Seules, des méthodes permettant de conserver le polymère à la paroi en évitant ou diminuant très considérablement leur perte par diffusion auraient quelques chances d'aboutir.

\section{Conclusions}

Parmi les multiples méthodes et systèmes proposés pour diminuer la résistance de frottement, parois souples actives ou passives, parois ondulées ou à "rugosité" positive, cháuffage pariétal, injection d'air, etc..., l'injection de solutions de polymères est la seule (avec les solutions micellaires et les suspensions de fibres) à montrer d'excellentes propriétés d'une manière répétitive, controlable et indiscutable. Les acquis théoriques et expérimentaux sont considérables et fournissent une base solide aux recherches futures qui doivent, à notre avis, être dirigées suivant deux axes. D'une part, il faudrait poursuivre les travaux dans le domaine des polymères et des fibres en recherchant des synergies possibles entre ces additifs. Ceci devrait être continué par des travaux portant sur la dégradation et l'autoréparation des composés macromoléculaires. D'autre part, dans le domaine concernant l'hydrodynamique externe, il faudrait pouvoir envisager des études sur l'exsudation des solutions de polymeres d'une manière contrôlée de façon à aboutir à des con- 
sommations au moins de deux ordres de grandeur inférieures à celles requises présentement.

Du point de vue des applications pratiques, un travail intense est à faire pour détecter des situations favorables à l'utilisation des polymères. Nous pensons que dans le cadre des motivations stratégiques, technologiques et économiques, il y a une place de choix pour cette mé. thode, surtout en tenant compte des situations nouvelles qui seront créées à l'avenir par l'augmentation constante des coûts de l'énergie.

\section{Remerciements}

Les auteurs tiennent à remercier les différentes organisations qui ont supporté financièrement leurs recherches. La DRET, la DGRST, le Ministère de l'Industrie et de la Recherche et le Ministère de l'Environnement et du Cadre de Vie ont montré beaucoup d'intérêt pour les travaux de la Société Bertin \& Cie, et lui ont apporté un soutien efficace et constant. D.H.F. tient à signaler que ses études sur l'injection de polymères ont été ren. dues possibles grâce à l'aide de l'Office of Naval Research (U.S Navy) et de la DRET, et aux possibilités qui lui furent offertes par Hydronautics Incorporated, Laurel, Maryland.

\section{Références}

[1] TOMS B.A. - Some observations on the flow of linear polymer solutions through straight tubes at large Reynolds numbers, Proc, lst Int. Rheol. Congr., North Holland Publ. Co, 1949.

[2] MYSELS K.J. - Early experiences with viscous drag reduction, A.I.Ch.E. Chem. Engng. Progr. Symposium Series, 67, $111,1971$.

[3] Hoyt J.W. - The effects of additives on fluid friction, Journal of Basic Engineering. Trans. ASME, Juin 1972.

[4] HOYT J.W. - Recent progress in polymer drag reduction, Polymères et Lubrification, CNRS, 1975.

[5] HOYT J.W. - Polymer drag reduction - A literature Survey 1975-76, Proc. Intnl. Conf. on Drag. Reduction, Cambridge, Aout 1977.

[6] DESLOUIS C. - La réduction de trainée hydrodynamique, Pour la Science, 7, Mai 1978.

[7] de LAGARDE B. - Réduction de frottement hydrodynamique par polymères hydrosolubles, Bertin \& Cie, Note Technique $n^{\circ}$ 73-Ce-03, 1973.

[8] FRUMAN D.H. et SULMONT P. - Réduction de la résistance de frottement d'une plaque plane dans les solutions de polymères; C.R. Acad. des Sciences 268, Juin 1969.

[9] VIRK P.S. - Drag reduction fundamentals, A.I.C.H.E. Journal. 21, 4, Juillet 1975.

[10] PETRY M. - Appareil permettant à volonté d'introduire un premier fluide dans un deuxième fluide ou d'isoler le premier fluide de second, Demande de Brevet d'Invention $n^{\circ} 7805946$.

[11] SELLIN R.H.J. et OLLIS M. - Polymer drag reduction in large pipes and sewers : results of recent field trials. Journal of Rheology, 24, 5, 1980.

[12] WELLS C.S. et SPANGLER J.G. - Injection of a drag reducing fluid into turbulent pipe flow of a Newtonian fluid, The Physics of Fluids, 10, 9, 1967.

[13] POREH M. - Drag reduction in a developing boundary layer with polymer solutions, Proc. 12th Int. Towing Tank Conf.,/Rome, Sept. 1969.

[14] TULLIS I.P. et RAMU K.L.V. - Drag reduction in developing pipe flow with polymer injection. Proc. Int. Conf. on Drag. Reduction, Cambridge, Sept. 1974.
[15] WU J. - Drag reduction in external flows of additive solution, Viscous Drag Reduction, Plenum Press, New York, 1969.

[16] WU J. et TULIN M.P. - Drag reduction by ejecting additive solutions into a pure-water boundary layer, A.S.M.E. Paper 72-FE-12, Mars 1972; aussi, Hydronautics, Inc. Techn. Report 353-7, Juin 1970.

[17] WU J. - Suppresed diffusion of drag-reducing polymer in a turbulent boundary layer, $J$. of Hydronautics, 6,1 , Janvier 1972.

[18] FRUMAN D.H. et TULIN M.P. - Diffusion of a tangential drag-reducing polymer injection on a flat plate at high Reynolds numbers, $J$. of Ship Research, 20, 3, Sept. 1976.

[19] LATTO B. et EL RIEDY K.F.O, - Diffusion of polymer additives in a developing turbulen $\imath$ boundary layer, $J$. Hydronautics, 10, 4, Octobre 1976.

[20] COLLINS D.J., GORTON C.W. - An experimental study of diffusion from a line source in a turbulent loundary layer, A.I.CH.E. Journal, 22, 3, May 1976.

[21] FRUMAN D.H. et TULIN M.P. - Drag reduction and diffusion accompanying thin sit injections of a drag reducing polymer on a flat plate at high Reynolds numbers, Hydronautics, Inc. Tech. Report 7101-3, Juin 1972.

[22] SEBAN P.A. - Heat transfer and effectiveness of a turbulent boundary layer with tangentiel fluid injection, Journal of Heat Transfer, Nov. 1960.

[23] POREH M. et HSU K.S. - Diffusion from a line source in a turbulent boundary layer, Intnl. J. of Heat and Mass Transfer, 14, 1971 .

[24] de LAGARDE B. - Injection parietale de corps macromoléculaires - Rapport de synthèse, Bert in \& Cie, Note Technique ${ }^{\circ} 73-\mathrm{Cm}-01,1973$.

[25] WU J., FRUMAN DH. et TULIN M.P. - Drag reduction by polymer diffusion at high Reynolds numbers, $J$. of Hydronautics, 12, 3, Juillet 1978 .

[26] VOGEL W.M. et PATTERSON A.M. - An experimental investigation of the effect of additives injected into the boundary layer of an underwater body, Proc. 5th Symposium on Naval Hydrodynamics, Bergen, Septembre 1964.

[27] FRUMAN D.H. et GALIVEL P. - Near field viscoelastic effects during thin slit drag-reducing polymer ejection, The effects during thin slit drag-reducing polymer ejection, Journal of Rheology, 24, 5, 1980.

[28] FRUMAN D.H. et GALIVEL P. - Anormalous effects associated with drag-reducing polymer ejection in to pure-water boundary layers, Symposium on Viscous Drag Reduction, Dallas, Novembre 1979 ; à paraître, Viscous Drag Reduction, AIA A Progress Series.

[29] FORESTER R.H., LARSON R.E., HAYDEN J.W. et WETZEL J.M. - Effects of polymer addition on friction in a 10-in.-diam. pipe, J. Hydronautics, 3, 1, Janvier 1969.

[30] DE LOOF J.P., de LAGARDE B., PETRY M. et SIMON A. - Pressure drop reduction in large industrial ducts by macromolecular additives, Proc. 2nd Intnl. Conf. on Drag Reduction, Cambridge, Aout 1977.

[31] FRUMAN D.H., LOISEAU G., NORGEOT G. et PIAU J.M. - Degradation of polyethylene oxide molecules in turbulent pipe flow, 1970 Intnl. Seminar on Heat and Mass Transfer in Rheologically Complex Fluids, Herceg Novi, 1970.

[32] BURGER E.D., CHORN L.G. et PERKINS T.K. - Studies of drag reduction over a broad range of pipeline conditions when flowing Prudhoe Bay crude oil. Journal of Rheology, $24,5,1980$.

[33] CHERON J., PETRY M. et RENARD D. - Role des macromolécules dans les écoulements d'eaux usées et/ou d'eaux pluviales, Ministère de l'Equipememt, Laboratoire Régional de l'Ouest Parisien, GSC 08, FAER I 0806 7.1979.

[34] POLLERT J. - Hydrotransport of fly ash-water mixture and drag reduction, Proc. Intnl. Conf. on Drag Reduction, Cambridge, Aout 1977.

[35] PETRY M. - Addition de macromolécules à l'eau des lances à incendie, Bertin \& Cie, Note Technique $n^{\circ} 77$ Cl 13, Décembre 1978.

[36] CANHAM H.J.S., CATCHPOLE J.P. et LONG R.F. Boundary layer additives to reduce ship resistance, Trans. Royal Inst. Naval Architects, 113, 3, Juillet 1971. 